\title{
A Gateway between the SCP-ECG and the DICOM Supplement 30 Waveform Standard
}

\author{
V Sakkalis ${ }^{1}, \mathrm{~F}_{\text {Chiarugi }}{ }^{1}$, S Kostomanolakis ${ }^{1}, \mathrm{CE}$ Chronaki ${ }^{1}, \mathrm{M}$ Tsiknakis ${ }^{1}$, SC Orphanoudakis ${ }^{1,2}$ \\ ${ }^{1}$ CMI-HTA, Institute of Computer Science, Foundation for Research and Technology - Hellas, \\ ICS-FORTH, Heraklion, Crete, Greece \\ ${ }^{2}$ Department of Computer Science, University of Crete, Heraklion, Crete, Greece
}

\begin{abstract}
Nowadays, the large-scale deployment of electronic health record systems and eHealth services has to face with a real multi-vendor environment. Even if each manufacturer supports an existing standard for communication and storage of ECG data, the large number of co-existing ECG standards is a major problem.

In this direction, an online service operating as a gateway between SCP-ECG, the European standard for communication and storage of resting ECGs, and the DICOM waveform standard (Supplement 30), a common format for the communication of time series data including vital signs and ECGs, has been developed.

The open availability of this service in the OpenECG portal is expected to facilitate digital ECG interoperability and contribute to the harmonization of ECG standards.
\end{abstract}

\section{Introduction}

Electrocardiography is one of the most important noninvasive diagnostic methods, which can be performed at a low cost and allows the early recognition of coronary heart disease. In today's distributed healthcare environment, ECG data are commonly acquired, stored and analysed using different formats and software platforms [1].

One of the major obstacles towards an integrated eHealth environment is the wide range of different mostly proprietary - digital storage formats that make the medical related files exchange between systems almost impossible. The practical and logistic implications of this restriction are obvious. More specifically, in the ECG domain, numerous new file formats offer different advantages against the older ones, because they are able to cover the known limitations of the oldest formats, but these ones are often widely accepted and comply with the exporting capabilities of the ECG acquiring devices. In practice, the integration in an existing system of a new ECG device supporting a different format from the currently managed formats (even if better) is a hard and time-consuming task.

There have been numerous approaches in the past, which attempt the conversion of different file formats and, as a result, commercial and cumbersome software packages were implemented. A step beyond was to move the same idea on the web by releasing specific web services that act as a gateway [2]. The latter idea inherits the dynamic, widely and highly accessible, easy to use environment of the web, while at the same time maintains platform independency.

Among the variety of ECG file formats, the SCP-ECG (the Standard Communications Protocol for ComputerAssisted Electrocardiography) is the European standard for communication [3] of resting ECGs and DICOM 3.0 Supplement 30 (Digital Imaging and Communications in Medicine - Waveform standard) [4] is a common format for the communication of time series data including ECGs. SCP-ECG is widely accepted even if limited to short term resting ECG, while DICOM turns out to be a one-way choice when it comes to file exchange and communication with numerous imaging modalities.

\section{Methods and materials}

The DICOM Waveform Interchange Standard (DICOM 3.0 Supplement 30 added in 2000) addresses the interchange of waveforms, including support for 12lead resting ECG data and time series data (Holter), time synchronization frame of reference, annotation capabilities and waveform synchronization within the imaging context. It can handle waveform storage of data independent from sampling frequency, amplitude and system sensitivity. DICOM is also particularly important in the context of the Integrating the Healthcare Enterprise initiative (IHE), which employs existing standards, such as DICOM and HL7 (Health Level Seven), for 
developing efficient and seamless workflows (i.e. integration profiles) using medical equipment and information systems from multiple vendors. The only drawback of DICOM is the complexity of the standard that requires a developer to have a prior knowledge of DICOM philosophy.

In 1993, the Comité Européen de Normalisation Technical Committee 251 (CEN/TC251) project team developed the SCP-ECG. The standard is relatively well established for the interchange, encoding and storage of digital ECG data. Patient demographics, ECG administrative data, as well as measurement and interpretation results, can be stored along with the ECG signal. The SCP-ECG standard specifies a communication protocol for computerized electrocardiography providing besides a detailed reference model and interchange format for ECG records, support for measurements, compression, and quality assurance. Although this standard is supported by some manufacturers of ECG equipment, the utilization of SCPECG has demonstrated mainly the following two disadvantages: it is short term resting ECG specific and there is only limited provision for annotation. On the other side the SCP-ECG standard provides a wellassessed support for lossless ECG compression and also for lossy ECG compression where the errors in the reconstructed signal are maintained within thresholds described in the standard itself sufficient to guarantee a correct re-interpretation of the ECG.

An overview of the main similarities and differences between the two standards are stated in table 1 .

Table 1. Comparison between SCP-ECG and DICOM standard.

\begin{tabular}{lcc}
\hline \multicolumn{1}{c}{ Standard Capabilities } & SCP-ECG & DICOM \\
\hline Information Model & $\mathbf{x}$ & $\checkmark$ \\
Representation Syntax & $\checkmark$ & $\checkmark$ \\
Transfer Mechanism & $\checkmark$ & $\checkmark$ \\
Not short term resting & $\mathbf{x}$ & $\checkmark$ \\
ECG specific & limited & $\checkmark$ \\
Annotation & & \\
Capabilities & $\checkmark$ & $\mathbf{x}$ \\
Support of lossless and & & \\
lossy compression & $\mathbf{x}$ & $\checkmark$ \\
Image Handling & & \\
\hline
\end{tabular}

In order to take advantage of the benefits of DICOM in an integrated multimedia patient record, where DICOM is used as middleware, the ability to convert any ECG time series into the DICOM waveform format is critical. This approach is easily feasible when the digital ECG time series are stored according to an established standard (i.e. SCP-ECG), but very hard and time- consuming when stored in a proprietary format (supposed that the proprietary format is disclosed).

The SCP-ECG to DICOM gateway has been implemented as middleware component and is also available as a web service. The online service receives SCP-ECG data files over an http connection and converts them to the DICOM waveform standard. The produced DICOM files can be saved as local DICOM files and are transmitted back at the requestor's demand, by supplying a reference link to the converted file. The whole process is illustrated in figure 1 . The converter is written in $\mathrm{C}++$ using a DICOM COM object that handles DICOM specific operations and formatting, as well as a library that parses the SCP-ECG formatted files. The web server is based on the Apache implementation. The whole process is transparent to the user.

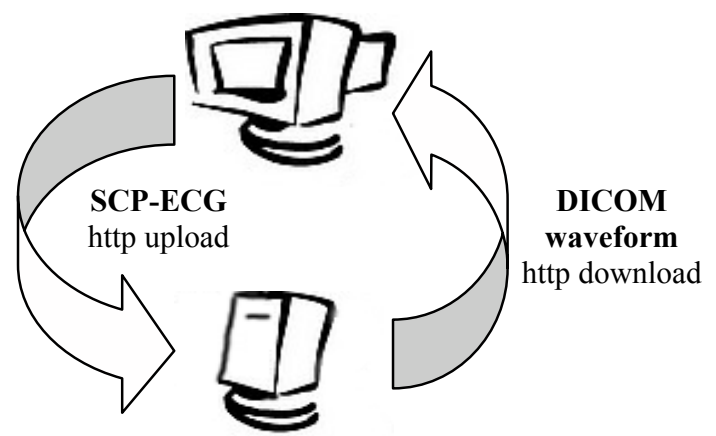

Figure 1. File exchange and conversion between the client $\mathrm{PC}$ and the server through the gateway conversion webservice.

In figure 2 the "Tools and Converters" section of the OpenECG members' area is shown. In this page the list of the available Tools and Converters is provided with their short description.

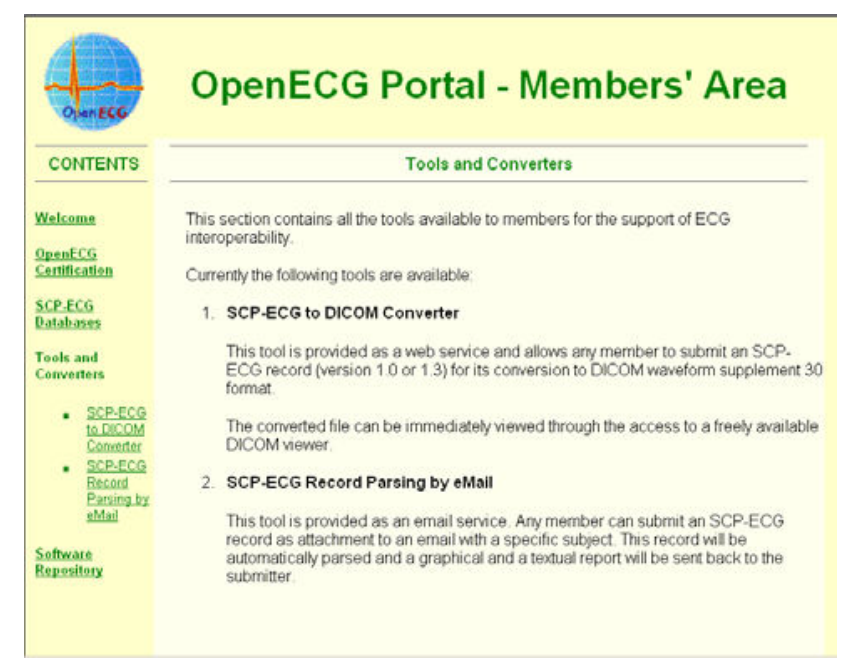

Figure 2. The OpenECG members' area with the description of the SCP-ECG to DICOM converter. 
Once the SCP-ECG to DICOM conversion service is accessed, in its entry page it is possible to browse the SCP-ECG file we need to convert. As soon as the file is chosen, the conversion is realized and its results can be accessed through the page shown in figure 3 .

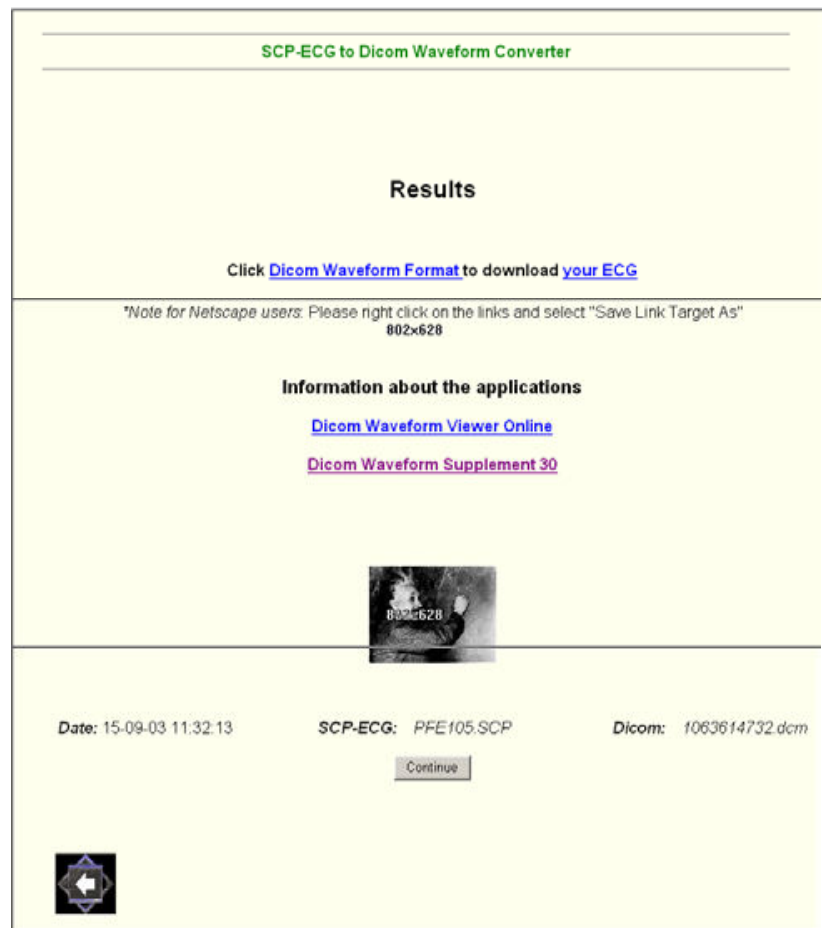

Figure 3. The results page of the OpenECG SCP-ECG to DICOM conversion service.

In this page the access to a DICOM waveform free viewer (Heart Institute - University of São Paulo) is available [5] and also the converted file in DICOM format can be downloaded by the requestor.

The main attention has been dedicated to the conversion of the ECG signals into the DICOM ECG time series. Only the main tags from section 1 are converted to DICOM tags, while many DICOM tags have been used for the storage of the ECG signal in its uncompressed format. Hence, backward conversion to the SCP-ECG format of DICOM waveform files that have been created by the gateway is possible (from an ECG signal point of view), using a lossless compression option provided by the SCP-ECG standard.

In this first version, the results of the ECG analysis and interpretation (reference beat, global measurements, diagnostic statements and lead dependent measurements) have not been considered. The conversion of this information, if its mapping is possible, will be studied and implemented in next versions of the converter.

Taking into account the wide diffusion of the DICOM standard and the fact that DICOM waveform viewers are freely available in the Web, while other ECG viewers (including SCP-ECG viewers) are still of limited availability; gateways among ECG standards are of particular value. One should point out that DICOM waveform viewers are currently available even for PDA platforms and java-enabled devices, as illustrated in figure 4 [6].

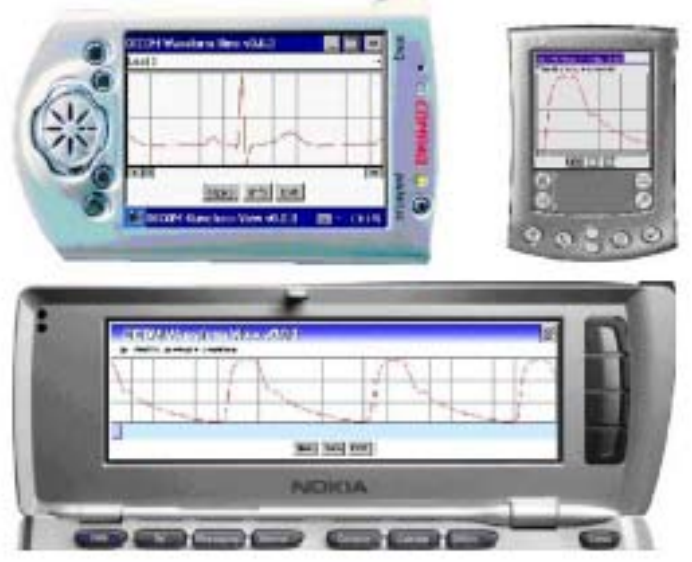

Figure 4. Mobile and PDA platforms capable of displaying DICOM waveforms including ECG files.

\section{Results}

The DICOM standard offers measurable benefits to digital ECG interchange, mainly because it integrates and homogenizes the whole medical environment. It provides a robust infrastructure mechanism for storing, retrieving and annotating time series and images. In addition, any associated reports and images may also be handled by the DICOM protocol and is possible to be reviewed on one local or remote workstation at the same time, as depicted in figure 5 .

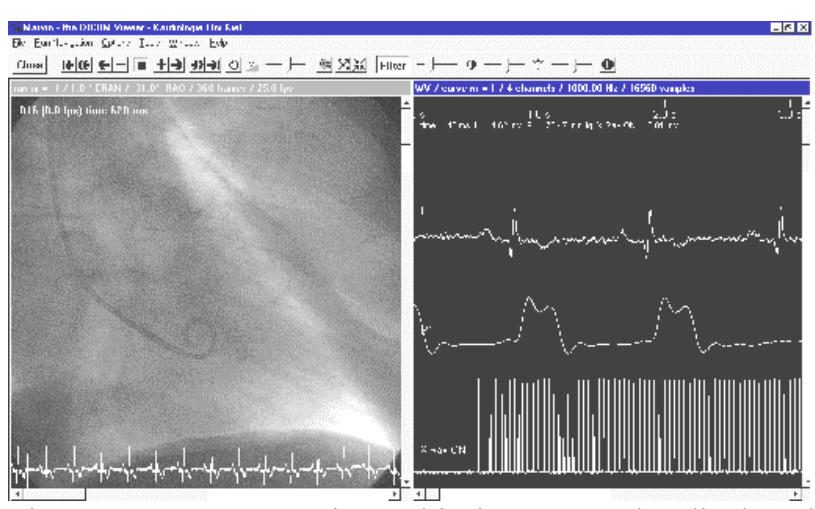

Figure 5. DICOM angiographic image can be displayed aside with waveform data, even in a synchronized mode.

In order to take advantage of the benefits of DICOM one should be able to transcode any digital ECG time series acquired in any digital format into the DICOM waveform format. In this context the gateway is of 
particular interest, since it acts as a middleware component that provides the necessary step needed towards interoperable medical applications, in the general clinical environment. Numerous SCP-ECG files either compressed or not, were successfully converted to DICOM. A freely available DICOM waveform viewer (Heart Institute - University of São Paulo) was used to ensure the correct conversion [5]. The conversion tool is included in the OpenECG portal (http://www.openecg.net) and is available only to the OpenECG members. This portal provides also additional tools to check the integrity and the conformance of the ECG files to the SCP-ECG standard; hence files can be checked prior to the conversion process.

The size of a SCP-ECG file depends on the duration of the signal, the number of measuring leads, the sampling rate and whether the data is compressed. In general the size for 10 seconds of resting ECG is small; hence the time consumed, in order to transfer the files over the network using the http protocol, is minimal. On the other hand, there is a big overhead added to the DICOM converted file since the data is kept uncompressed and the DICOM header is included on top of the file. Furthermore, the structured way of writing DICOM files that include coded entry sequences creates larger files. Despite the fact that the converted files can be more than ten times larger in size than the original ones, they are still limited in size and can be quickly transferred over the Internet. The converted files that we have tested never exceeded the limit of $200 \mathrm{kB}$ in size.

\section{Conclusions}

The discussed topics and applications conclude to the fact that it is possible to realize seamless workflow scenarios in a medical environment by utilising freely available converters in cases where non-standardized formats are present. Of course converters between existing and well-established standards can be of further value, being able to provide a solution for all medical devices supporting these standards and not only for a single manufacturer.

Future work will be focused on the enrichment of this web service with web-enabled components specialized for viewing ECG files. Measuring capabilities will also be provided.
The use of these gateways as part of ECG viewing components with measurement and serial comparison capabilities are likely expected to be a breakthrough for eHealth applications that incorporate digital ECGs.

\section{Acknowledgements}

The work reported in this paper was supported in part by the OpenECG project (IST-2001-37711).

\section{References}

[1] Värri A, Kemp B, Penzel T, Schlögl A. Standards for biomedical signal databases. IEEE Eng Med Biol Mag. 2001 May-Jun;20(3): 33-7.

[2] Kostomanolakis S, Chronaki C, Tsiknakis M, Orphanoudakis SC. DicomGW: DICOM functionality as middleware. Poster Presentation in the ECR 2000, Scientific Exhibit Compurad/EURORAD, Vienna, Austria, 5-10 March 2000.

[3] ENV 1064 standard communications protocol for computer-assisted electrocardiography. European Committee for Standardisation (CEN), Brussels, Belgium, 1993.

[4] DICOM Suppl. 30, Waveform Interchange, Nat. Elect. Manufacturers Assoc.: ARC-NEMA, Digital Imaging and Communications, NEMA, Washington D.C., 1999.

[5] http://www.excelmedical.com/waveforms/Viewer/DicomWaveformViewer. htm. Division of Informatics, Heart Institute - University of São Paulo, Brazil.

[6] Kroll M, Melzer K, Lipinski HG. Accessing DICOM 2D/3D-Image and Waveform Data on Mobile Devices. Mobile Computing in Medicine 2002: 81-86, Heidelberg, Germany, 2002.

Address for correspondence.

Vangelis Sakkalis

CMI-HTA,

Institute of Computer Science,

Foundation for Research and Technology - Hellas,

P.O. Box 1385 Vassilika Vouton,

GR 71110 Heraklion, Crete

Greece

sakkalis@ics.forth.gr 